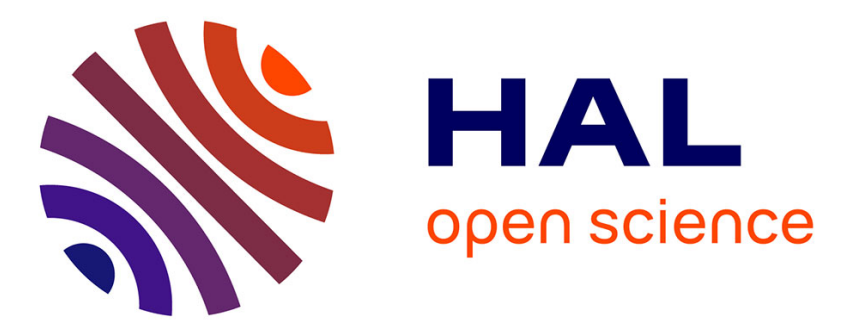

\title{
Diegetization: An Approach for Narrative Scaffolding in Open-World Simulations for Training
}

Kevin Carpentier, Domitile Lourdeaux

\section{To cite this version:}

Kevin Carpentier, Domitile Lourdeaux. Diegetization: An Approach for Narrative Scaffolding in Open-World Simulations for Training. 7th International Conference on Interactive Digital Storytelling, Alex Mitchell, Nov 2014, Singapour, Singapore. pp.25 - 36, 10.1007/978-3-319-12337-0_3 . hal01095974

\section{HAL Id: hal-01095974 \\ https://hal.science/hal-01095974}

Submitted on 16 Dec 2014

HAL is a multi-disciplinary open access archive for the deposit and dissemination of scientific research documents, whether they are published or not. The documents may come from teaching and research institutions in France or abroad, or from public or private research centers.
L'archive ouverte pluridisciplinaire HAL, est destinée au dépôt et à la diffusion de documents scientifiques de niveau recherche, publiés ou non, émanant des établissements d'enseignement et de recherche français ou étrangers, des laboratoires publics ou privés. 


\title{
Diegetization: an approach for narrative scaffolding in open-world simulations for training
}

\author{
Kevin Carpentier and Domitile Lourdeaux \\ Heudiasyc - UMR CNRS 7253, \\ Université de Technologie de Compiègne, 60200 Compiègne, France \\ \{kevin.carpentier, domitile.lourdeaux\}@hds.utc.fr \\ http://www.hds.utc.fr
}

\begin{abstract}
The use of storytelling for learning is widely approved and encouraged. Yet, in virtual environments for training, there are difficulties to build a story when there is no global control over the course of events. We present in this paper an approach called diegetization. Supported by structuralist narrative theories, this approach aims to dynamically match a sequence of events with sequence of narrative patterns. The pedagogical prescriptions are then extended to consolidate the recognized narrative sequences. The process uses semantic models to benefit from pattern matching algorithm and deep inferences. The proposition was implemented in the HUMANS platform and applied to a scenario for training in high-risk activities.
\end{abstract}

Keywords: virtual training, interactive storytelling, constraint satisfaction problem, structuralism, semiotic, semantic representation

\section{Introduction}

\subsection{Context}

This paper describes an approach for framing a sequence of events in a virtual simulation to comply with narrative constraints. We focus our studies on virtual environments for training (VET) and especially on open-world simulations. Open-world simulations offers a high freedom of action to enable situated learning. In order to mimic real work situations, such simulations are populated with autonomous characters upon which no direct control can be enforced. Such a design permits the emergence of unexpected scenarios without authoring effort. Pedagogical control is added by taking into account expert knowledge from trainers or by joining an Intelligent Tutoring System (ITS) to the VET. Trainers and ITS can monitor the simulation by dynamically prescribing relevant learning situations. Then, a scenario manager is responsible for guiding the simulation toward the prescribed situations. This architecture enables the generation of a highly personalized pedagogical scenario which is experienced by the user in the simulation. Yet, trainers are not storytelling experts and an ITS does not always consider narrative aspects. Without narrative control, pedagogical scenarios lack story continuity and tension management. Our purpose is to ensure 
that events taking place in the simulation, occurring autonomously or resulting from a prescription, tell an appealing story. A first difficulty lies in recognizing attractive plot lines in a sequence of event. Another difficulty is to add narrative aspects to trainer's prescriptions without prejudice to the pedagogical intent. In order to achieve this, we propose to consider the problem as a constraint satisfaction problem. The originality lies in the semantic representation of narrative constraints to allow easy authorship and deep reasoning.

To illustrate our work, we propose here a simple example of training of operators on hazardous-matter loading. A trainer prescribes a pedagogical situation where the learner would have to handle a hazardous-matter leak on a tank. Such an incident usually results from human neglect. Therefore, the prescription involves a responsible and a leaking valve. The prescription could be instantiated by picking any character and any tank in the virtual environment. However, by selecting these entities according to narrative rules, we can create continuity upon the sequence of events. A storyworld is gradually scaffolding each time a prescription is enriched with narrative content.

We named this approach "diegetization" in reference to the authoring process of building the diegesis [1]. The diegesis is the universe in which the story take place. In the next section of this paper, motivations for the choice of a narrative construction are presented. Then, a brief review of related works is presented. The process of diegetization and its application are described in section 3 and 4. The last section concludes this paper with discussions and perspectives.

\subsection{Narrative for learning}

A first argument for the use of narrative in learning context is quite pragmatic. Indeed, as narrative is a natural expressive form [2], it can be presented through different modalities. It can be told through text or speech, shown through pictures or, as in this case, experienced through an interactive simulation. Moreover, narrative is a support for cognitive activities. Through narrative, learners are engaged in active thinking and are supported in their process of meaning construction [3]. This helps learners to organize knowledge and to develop cognitive abilities [4]. Narrative can also be instrumented to build motivation. Motivation is most significant to ease learning processes in pedagogical activities [5] in many ways. First, a storyline can arouse learners' curiosity. By taking into account their behaviours, an interactive narrative reinforces the learners' feeling of control in the simulation. Moreover, a storyworld is where fantasy can take place: learners can identified themselves with characters depicted through the story. Finally, narrative also provides interpersonal motivation through cooperation and competition between the user and the other characters depicted in the storyline. Besides, a strong motivation comes from recognition: it happens when the learners are rewarded for their good behaviours.

As storytelling proves to be particularly efficient for learning and training, we aim to add a narrative layer upon the pedagogical prescription over the scenario. The definition of a quality criteria of a narrative is therefore fundamental to assess the quality of the content generated by the system. An engine for building 
narrative has to offer variability, thus same kind of situations can be told through different stories. It also has to be resilient to cope with whatever actions the autonomous characters or users may perform against the storyline. However, the production of a VET is a very time-consuming task. The addition of narrative to a VET should be easy and should require a minimal amount of authoring. The work presented in this paper try to tackle the issue of balancing quality and resilience without compromising variability and minimal handmade authoring.

\section{Related Work}

First attempts of story generation make the narrative emerges from the mechanical execution of world rules $[6,7,8]$. [9] argues that such systems, qualified as world-simulation based story generation lack the creative process of the author. She proposed an author-intent-based process: the author build a story around a sequence of events, therefore he/she is able to add/modify/remove characters, locations or plot points in order to create richer stories. [10] pushed this notion further and proposed an engine that simulates an author's behaviour in a two-phased generation process: an engagment stage to explore every possibilities and a reflection stage to ensure coherency. We think a similar approach can be adapted to our dynamic context to foster the story quality.

Recent works on story generation are usually pinned on a spectrum ranging from strong story approaches to strong autonomy approaches depending on the amount of global control [11]. In strong autonomy approaches [12], virtual characters act independently without any coordination. Yet, the story emerges from the individual decisions of the virtual characters. However, the lack of coordination between characters infringes the global quality of stories. In $[13,14]$, a central agent controls the simulation to ensure that the unfolding of events follows a specific path. Thanks to this control, it is possible to generate stories of high quality. Yet, as virtual characters are being supervised their behaviours might seem incoherent. To tackle this issues, some works attempt to balance global control with autonomous behaviours. In [15], the world is populated by semi-autonomous characters: they act freely until the Automated Story Director gives them an order. Even if the transition between the autonomous stage and the controlled stage is subtle, the switch in the personality might seem incoherent to the user. FACADE [16] also relies on a hybrid approach: virtual characters act autonomously to satisfy a goal proposed at a higher level by a drama manager which handle the coherency. Unfortunately, this kind of approach requires a huge amount of authoring.

Whatever approach is used, an interactive storytelling system embeds preferences about narrative aspects. Such preferences can either be explicit or implicit. Explicit preferences are specific plot points which are preferred over other because they are mandatory for the story continuity or because they induce a particular tension effect [17]. Implicit preferences allow a more subtle control by putting constraints on properties of the scenario. For instance, in IDTENSION 
[18], characters' actions are selected to maximise narrative criterion such as the cognitive load or the level of conflict.

The work presented in this paper is another attempt to balance strong story approach with emergent behaviours. The simulation in which the scenario take place is executed through a character-based approach: the VET is populated by autonomous characters upon which no control is possible. However, they were designed to reproduce human behaviours, they do not comply to narrative constraints. Therefore, global supervision is necessary to manage tension, to assure narrative continuity and to provide an appealing story.

\section{Approach}

\subsection{Narrative Theory}

[19] defines a narrative as the recounting of a sequence of events that have a continuant subject and constitute a whole. This definition constitutes a lower bound to what has to be achieved: the events taking place in the simulation must have a continuant subject and be holistically understandable. Since classical era, the minimal steps of a story have been canonized. Grammatical views of narrative were proposed to analyse existing texts [20, 21]. They were used more recently to generate narrative [22]. A purely grammatical approach is too restrictive and would hardly fit the resilience objective. Yet, [23] suggested the concept of grammaticality which rests upon three principles:

1. A narrative is better than another one if it is more compliant to a grammar than the other one.

2. Narrative structures must respect grammar ordering.

3. High-level constraints are more important than low-level constraints.

These considerations focus on the sequence of a narrative but struggle to depict the structure of one element of the sequence. Building his theory upon Greimas' actancial model $[24,25]$ proposed a more abstract model consisting of the following elements:

- Functions are relevant actions in regard of the story progression- Example: Saving the World

- Roles are functions grouped together - Example: The Savior

- Actants are abstract entities entitled with one or many roles - Example : The Protagonist

- Actors are concrete instances of actants - Example: Eric

This structuralist approach offers a framework to describe abstract story organizations. Therefore, it can be used to model stereotypical narrative structures such as the dramatic situations listed by [26] or [27].

These thoughts from various semioticians have guided the design of the diegetization process. First, we design a model, inspired by Bremond's grammar, to inform abstract narrative patterns. Graph matching algorithms recognize 
these narrative sequences in the unfolding of events. Then, techniques for constraint satisfaction problems are used to select and instantiate patterns fulfilling the provided pedagogical goal. A quality criteria assess the best pattern among the possibilities. This process illustrated in figure 1 is explained in following sections.

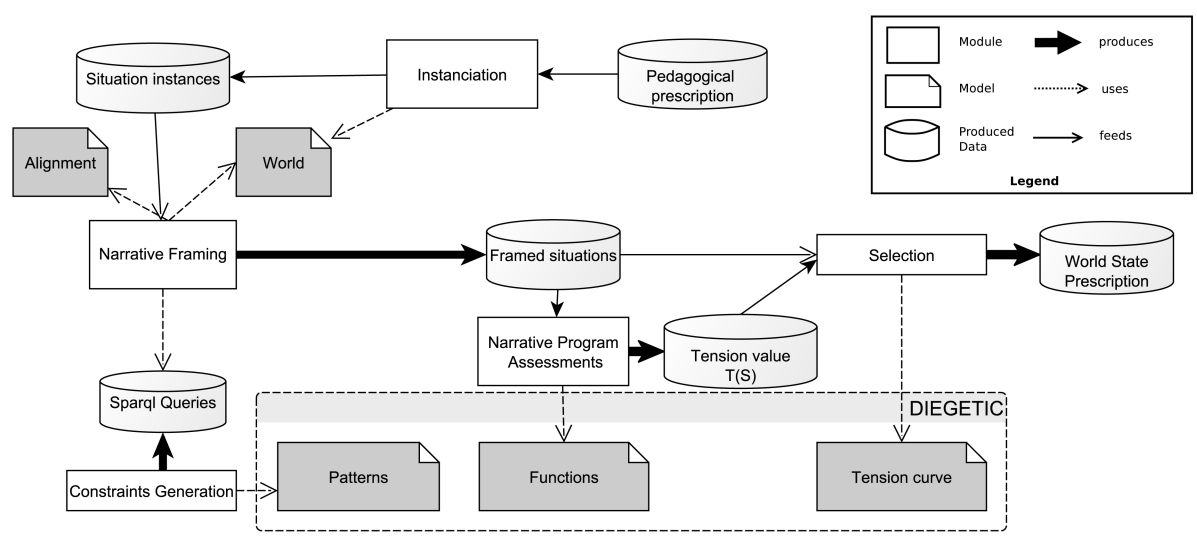

Fig. 1. Schematic view of the diegetization process

\subsection{Models}

The diegetization process requires to reason about two kinds of knowledge: knowledge about the world depicted by the simulation and knowledge about narrative patterns. For the sake of modularity, these two kinds of knowledge are informed in two different models. Consequently, the model of narrative patterns can be used with different models of the world which makes the approach fully domain-independent. For both models, the use of semantic representation allow for rich inferences about object capabilities and their evolution. The WorLD model and the DIEGETIC model are ontologies written in OwL/RDF language. Therefore, they benefits from owL semantic inferences and can easily be queried using SPARQL for graph pattern matching.

WORLD Model. The WorLD model describes the world simulated by the virtual environment. It especially informs knowledge about the agents that populate the virtual world, the objects with which the user can interact and possible actions he/she can trigger. The WORLD model is written by experts of the domain of application and do not consider any narrative aspects. It is fully domain-dependent. 
DIEGETIC Model. The Diegetic model is directly inspired by Bremond's grammar. It describes high-level entities to enable the specification of narrative structures:

- Element described meaningful entities within a narrative context. There are two types:

- Role: a set of capabilities -Example: The Traitor

- Actant: an aggregation of Role - Example : Antagonist

Elements can either be impersonated by characters, concrete objects (items, locations, etc.) or abstract objects (life, love, etc.).

- Function: a relationship linking an Element to another one or to a primitive type (boolean, float, string, etc.). They can be involved in two kind of expressions:

- Constraints are nomological constraints describing the state in which an Element should be to make a narrative structure possible - Example: (?x is-a :Antagonist) (?x :has-ally ?y)

- Properties are relationships checked by a narrative structure - Example: (?x is-a :Treator) (?y is-a :Protagonist)(?x :harm ?y).

: is-a,: has-ally and :harm are examples of functions. Functions are associated with :

- an intrinsic-significance (IS) value which measures the importance of the object for the subject of the relation on a zero to one scale. Example: the :has-ally function has an IS 0.7. It means that the modification or the removal of the function between ?x and ?y is quite important for ?x.

- an extrinsic-significance (ES) value which describes the importance of the function in a narrative structure. Example: the : harm function has an ES value of 0.95. It means this function is fundamental for the pattern in which it is expressed to be meaningful.

- Pattern is a specific set of functions between roles, it is also referred as narrative structure.

- Path describes the preferable transition between two Patterns - Example: Treason $\rightarrow$ Remorse.

The DIEGETIC model is fully domain-independent and is used to describe any narrative pattern. It can be filled by storytelling experts, yet, we plan to write a canonical library of narrative patterns inspired by Polti's dramatic situations [26]. A sequence of narrative pattern written in the DIEGETIC representation is shown in figure 2 .

\subsection{Instantiation}

Periodically, the pedagogical module produces a pedagogical prescription. It depicts a world state which should be encountered by the learner. Situations are formalized through rdf graphs consisting in fragments of a WoRLD model. Such fragment generally includes both conceptual descriptions such as (?anyvalve :has-state :leaking) as much as instantiated descriptions such as (:Valve01 :has-state :leaking). Graphs including abstract concepts need to be instantiated by referring to the up-to-date world state. A graph can be instantiated in various ways (figure 3 ). 


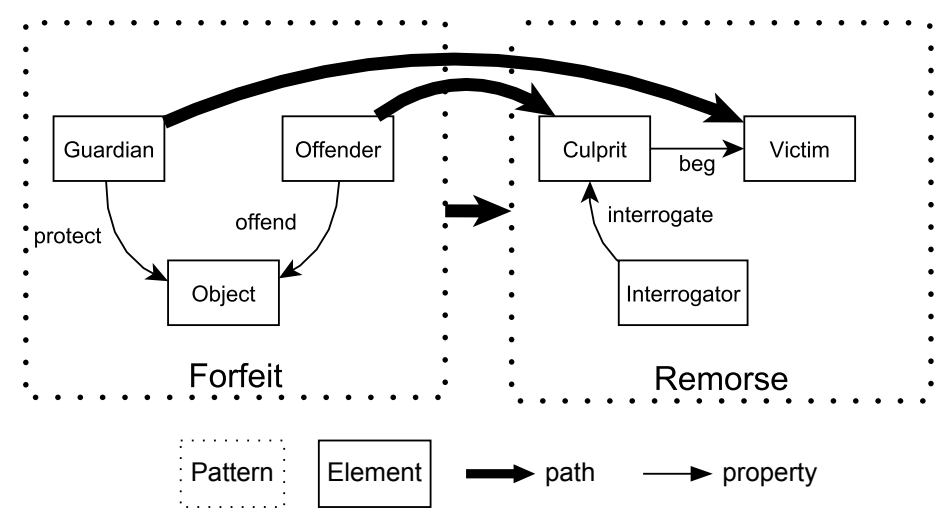

Fig. 2. A sequence of narrative patterns in the DiEGETIC model

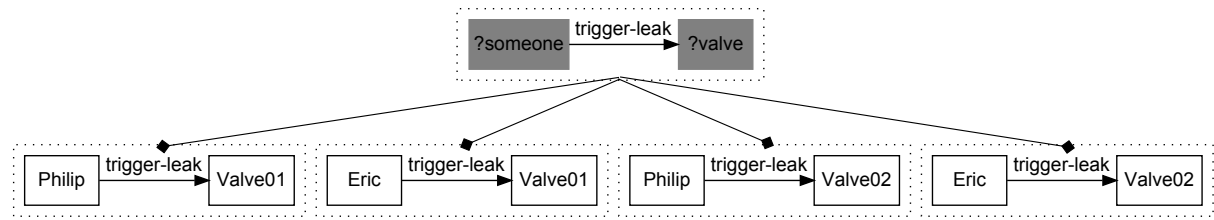

Fig. 3. Instantiation

\subsection{Narrative Framing}

For each instantiated situation, a possible world is created by assuming that the situation is true in the current world state. The narrative framing process searches possible world to recognize narrative patterns which are consistent with the storyline. This is a three-stage process: alignment, exploration and consistency-checking.

Alignment. The world state, which is fully domain-dependent, must be translated in terms of generic diegetical functions e.g. either properties or constraints. As both models are expressed through ontological representations, we use alignment rules to infer existing diegetical functions in the current world state. These rules are common-sense knowledge and can be written by pedagogical experts. For instance, a simple rule infers that characters involved in cooperative work (WORLD model) are mutual allies (DIEGETIC model).

Exploration. SPARQL queries are generated from the description of the patterns in terms of functions. Each possible world, describing an alternate world state, is interrogated by these queries. The results from these queries feature all possible instantiations of the pedagogical prescription fitting into a narrative structure. Queries are generated so that their results provide a mechanism 
similar to late commitment[12]: a pattern frames a situation if it finds no contradictions. A framed situation involves the entities of the initial prescription. Beside, it also involves other entities needed to fill all the elements in the pattern. A framed situation is formalized through a graph $S$ whose nodes are elements and whose arcs are functions. At this stage, each possibility is explored regardless of the coherence between the pattern and past events.

Coherency-checking. This stage aims to assure coherency of the whole story taking place since the beginning of the simulation. It checks the existence of a path between current situations and previous ones. Inconsistent situations are dismissed. In the case where no situations survive this stage, trade-off are performed: constraints are relaxed by deleting less-valued properties and a new cycle of exploration/consistency-checking is performed. The coherency-checking stage ensures that the narrative elements remain consistent. For instance, an ally will remain an ally throughout the plot line unless a pattern of treason make it otherwise.

\subsection{Narrative Program Assessment}

[24] proposed a formal description of the evolution of the entities in a story through narrative program. A narrative program is the gain or the loss of a function $R$ between a subject $S_{2}$ and an object $O$ when it is considered by an observer $S_{1}$. In other words, a narrative program describes a shift in the relationship between $S_{2}$ and $O$ as perceived by $S_{1} . S_{1}, S_{2}$ and $O$ can be assimilated to diegetical elements (objects, characters, places, etc.).

We proposed to use narrative program to measure the evolution of dramatic tension during the sequence of events. The narrative program assessment computes the value of the tension generated between two simulation steps by using the intrinsic and extrinsic significance defined in section 3.2. An instrinsic value between $S_{1}$ and $S_{2}$, noted $I\left(S_{1}, S_{2}\right)$, is the maximum of the products of the intrinsic-significance of the function in all the possible paths between $S_{1}$ and $S_{2}$. $E(R)$ is the extrinsic-significance of the function $R . T\left(S_{1}\right)$ is the tension for the subject $S 1$. Its value is the sum of all narrative programs $(N P)$ in which it is involved :

$$
T\left(S_{1}\right)=\sum_{(s, r, o) \in N P\left(S_{1}\right)} I\left(S_{1}, s\right) *(1+I(S 1, o)) * E(r) .
$$

We propose to use the value $T(U$ ser $)$ where $U$ ser is the entity representing the user in the knowledge base in order to monitor the dramatic tension he/she perceives.

\subsection{Decision using a quality criteria}

Each framed situation $\mathcal{S}$ is evaluated according to three criterion: 
- $O(\mathcal{S}) \in[0,1]$ is a measure of adequacy between opening needs and opening opportunities. The opening measures the number of narrative path that can follow the current step. In the beginning of the simulation, the opening needs are high to maximise the possibilities of diegetization. Such constraints can be relaxed when the session reaches its end.

$-T(\mathcal{S}) \in[0,1]$ is the ratio between the estimated tension of $S$ and the expected value of tension according to a pre-defined curve.

- $G(\mathcal{S}) \in[0,1]$ is an evaluation of the grammaticality of a framed situation. It takes into account the trade-off made on the constraints during the exploration/consistency-checking cycle.

The narrative utility of $\mathcal{S}$ according to the current step of simulation is $U(\mathcal{S})=O(\mathcal{S})+T(\mathcal{S})+G(\mathcal{S})$. The situation $\mathcal{S}$ with the highest $U(\mathcal{S})$ is selected to be the world state prescription.

\section{Results}

\subsection{Implementation}

We implemented the diegetization approach in Java using the JenA Framework [28]. It communicates as an agent in the multi-agent system of the Humans platform [29].

The Director module introduced by [30] is used to lead seamlessly the simulation toward the world state prescriptions. At the moment, the story is only represented by mimetic means. The user experienced the story through the actions of the characters and through the different event taking place. Further research will consider possibilities to foster narrative understanding such as dia$\log$ generation and extra-diegetic indication. At the moment, a selection among the thirty-six Polti's dramatic situations [26] have been informed as pattern in the Diegetic model.

\subsection{Application}

The approach have been applied on a very delimited use-case: virtual training for loading hazardous-matter in oil depot. We design a WorLD model with the help of domain expert for this application. It includes four characters. Bob is the user's avatar. Philip is one of Bob's co-workers. Eric works in a different team. Paul is their supervisor. Philip, Eric and Paul are controlled by autonomous characters. Workers have to perform hazardous-matter loading on a tank through valves. Leaks can occur due to carelessness in the procedure.

For the sake of the demonstration we only present three steps in the simulation with two different tension curves (figure 4). The first condition involves a classical aristotelian tension arc, in which the climax, occurring at the second step has a medium tension value. In the second condition, the climax has a high tension value and is followed by a step with a medium tension value. 


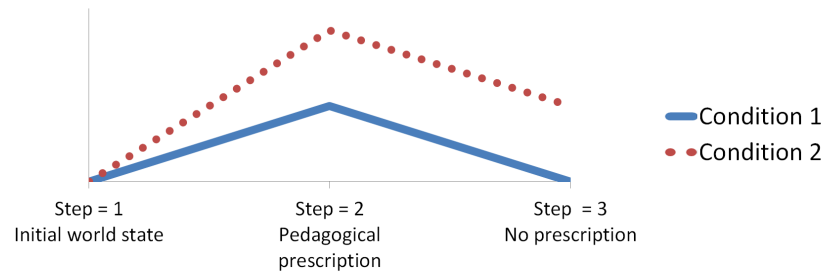

Fig. 4. Curve of tension for condition 1 and 2

In both conditions, a first diegetization procedure is run after the pedagogical module from the Humans platform [31] has issued a prescription : (?someone :trigger-leak ?valve). A new diegetization procedure without specific pedagogical goal is issued after the resolution of the previous situation. Partial results

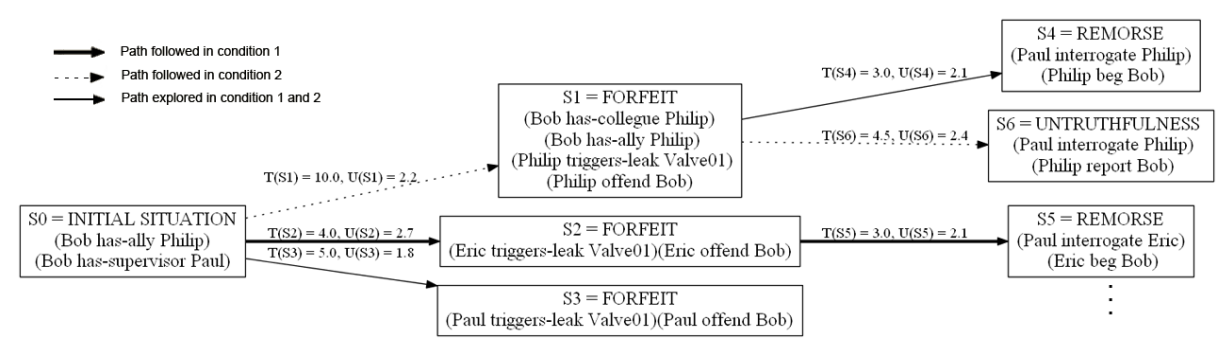

Fig. 5. Results of the diegetization process in condition 1 and 2

of the execution are presented in figure 5 .

In the first step, the prescription issued by the pedagogical module is instantiated for each characters. In particular these situations can be framed by $S 1, S 2$ and $S 3$ (other possibilities were not presented in the figure). In the condition 1, $S 2$ is preferred to $S 1$ according to the tension adequacy criteria. $S 3$ is dismissed in both condition because it lacks opening opportunities.

In both $S 1$ and $S 2$ Bob is offended by another worker. In $S 1$, Bob is offended by someone who is his ally. The intrinsec-significance of offend and has-ally are taken into account to compute $T(B o b)$ in the narrative program assessment. $S 1$ is more tense than $S 2$. It is explained by the fact that it is harder to be offended by someone you are supposed to rely on. Thanks to quality evaluation, $S 1$ fits with the first tension curve whereas $S 2$ fits with the second tension curve. $S 1$ is followed by $S 4$ and $S 6$. The extrinsec-significance of beg is quite high. Therefore, to comply with the expected tension in condition $1, S 6$ is preferred over $S 4$. In the first step, a role is given to elements of the simulation (characters in this case). It is gradually strengthened and enriched throughout the scenario to scaffold the story universe in accordance with a predefined tension curve. 


\section{Conclusion}

Our work on pedagogical orchestration of training simulation leads us to consider using narrative as a motivational factor. Yet, the simulation taking place in an open-world, the control possibilities are quite seldom. To tackle this difficulty, we proposed the process of diegetization which relies on the recognition and on the scaffolding of narrative structure. Our propositions were implemented in the Humans platform and tested on a small use-case. Results are in agreement with the objectives. In the short term, we will apply the proposition to a real use-case in training for risk prevention. Experiments on the perception and the opinion of the generated storylines by the user will be conducted. Thanks to this study, we will be able to consider the best possibilities to foster story understanding without breaking the feeling of immersion of the user.

\section{Acknowledgements}

This work was made possible thanks to the funding of ANR project: NIKITA (Natural Interaction, Knowledge and Immersive systems for Training in Aeronautic).

\section{References}

1. Odin, R.: De la fiction. De Boeck Supérieur (September 2000)

2. Bruner, J.S.: Acts of meaning. Harvard University Press (1990)

3. Dettori, G., Paiva, A.: Narrative learning in technology-enhanced environments. In Balacheff, D.N., Ludvigsen, D.S., Jong, D.T.d., Lazonder, D.A., Barnes, D.S., eds.: Technology-Enhanced Learning. Springer Netherlands (January 2009) 55-69

4. Schank, R.C.: Tell me a story: Narrative and intelligence. Northwestern University Press (1995)

5. Malone, T.W., Lepper, M.R.: Making learning fun: A taxonomy of intrinsic motivations for learning. Aptitude learning and instruction 3(3) (1987) 223-253

6. Klein, S., Aeschlimann, J.F., Balsiger, D.F.: Automatic novel writing: A status report. Wisconsin University (1973)

7. Meehan, J.R.: TALE-SPIN, an interactive program that writes stories. In: IJCAI. (1977) 91-98

8. Bringsjord, S., Ferrucci, D.: Artificial intelligence and literary creativity: Inside the mind of brutus, a storytelling machine. Psychology Press (1999)

9. Dehn, N.: Story generation after TALE-SPIN. In: IJCAI. Volume 81., Citeseer (1981) 16-18

10. Pérez, R.P., Sharples, M.: MEXICA: A computer model of a cognitive account of creative writing. Journal of Experimental \& Theoretical Artificial Intelligence 13(2) (2001) 119-139

11. Mateas, M., Stern, A.: Towards integrating plot and character for interactive drama. (2000)

12. Swartjes, I., Kruizinga, E., Theune, M.: Let's pretend i had a sword. In: Proceedings of the 1st Joint International Conference on Interactive Digital Storytelling: Interactive Storytelling, Springer-Verlag (2008) 264-267 
13. Mott, B.W., Lester, J.C.: U-director: a decision-theoretic narrative planning architecture for storytelling environments. In: Proceedings of the fifth international joint conference on Autonomous agents and multiagent systems. AAMAS '06, New York, NY, USA, ACM (2006) 977-984

14. Young, R.M., Riedl, M.O., Branly, M., Jhala, A., Martin, R.J., Saretto, C.J.: An architecture for integrating plan-based behavior generation with interactive game environments. Journal of Game Development 1(1) (2004) 51-70

15. Riedl, M.O., Stern, A., Dini, D., Alderman, J.: Dynamic experience management in virtual worlds for entertainment, education, and training. International Transactions on Systems Science and Applications, Special Issue on Agent Based Systems for Human Learning 4(2) (2008) 23-42

16. Mateas, M., Stern, A.: Façade: An experiment in building a fully-realized interactive drama. In: Game Developers Conference (GDC'03). (2003)

17. Porteous, J., Cavazza, M., Charles, F.: Applying planning to interactive storytelling: Narrative control using state constraints. ACM Transactions on Intelligent Systems and Technology (TIST) 1(2) (2010) 10

18. Szilas, N.: A computational model of an intelligent narrator for interactive narratives. Applied Artificial Intelligence 21(8) (2007) 753-801

19. Prince, G.: A dictionary of narratology. U of Nebraska Press (2003)

20. Propp, V.I.: Morphology of the Folktale. University of Texas Press (June 1968)

21. Todorov, T.: La grammaire du récit. Langages (1968) 94-102

22. Peinado, F., Gervás, P.: Evaluation of automatic generation of basic stories. New Generation Computing 24(3) (2006) 289-302

23. Beck, U.: La linguistique historique et son ouverture vers la typologie. Editions L'Harmattan (1976)

24. Greimas, A.J.: Sémantique structurale : recherche et méthode. Larousse edn. (1966)

25. Bremond, C.: Logique du récit. Éditions du Seuil (1973)

26. Polti, G.: Les 36 situations dramatiques. Éd. DV" Mercure de France" (1895)

27. Souriau, t.: Les deux cent mille Situations dramatiques. Flammarion (1950)

28. Apache Jena, https://jena.apache.org

29. Lanquepin, V., Lourdeaux, D., Barot, C., Carpentier, K., Lhommet, M., Amokrane, K.: HUMANS: a HUman models based artificial eNvironments software platform. In: Proceedings of the Virtual Reality International Conference (VRIC 2013), Laval, France (2013)

30. Barot, C., Lourdeaux, D., Lenne, D.: Dynamic scenario adaptation balancing control, coherence and emergence. In: Proceedings of ICAART'13: International Conference on Agents and Artificial Intelligence. Volume 2., Barcelona, Spain (2013) 232-237

31. Carpentier, K., Lourdeaux, D., Mouttapa-Thouvenin, I.: Dynamic selection of learning situations in virtual environment. In: Proceedings of ICAART'13: International Conference on Agents and Artificial Intelligence. Volume 2., Barcelona, Spain (2013) 101-110 